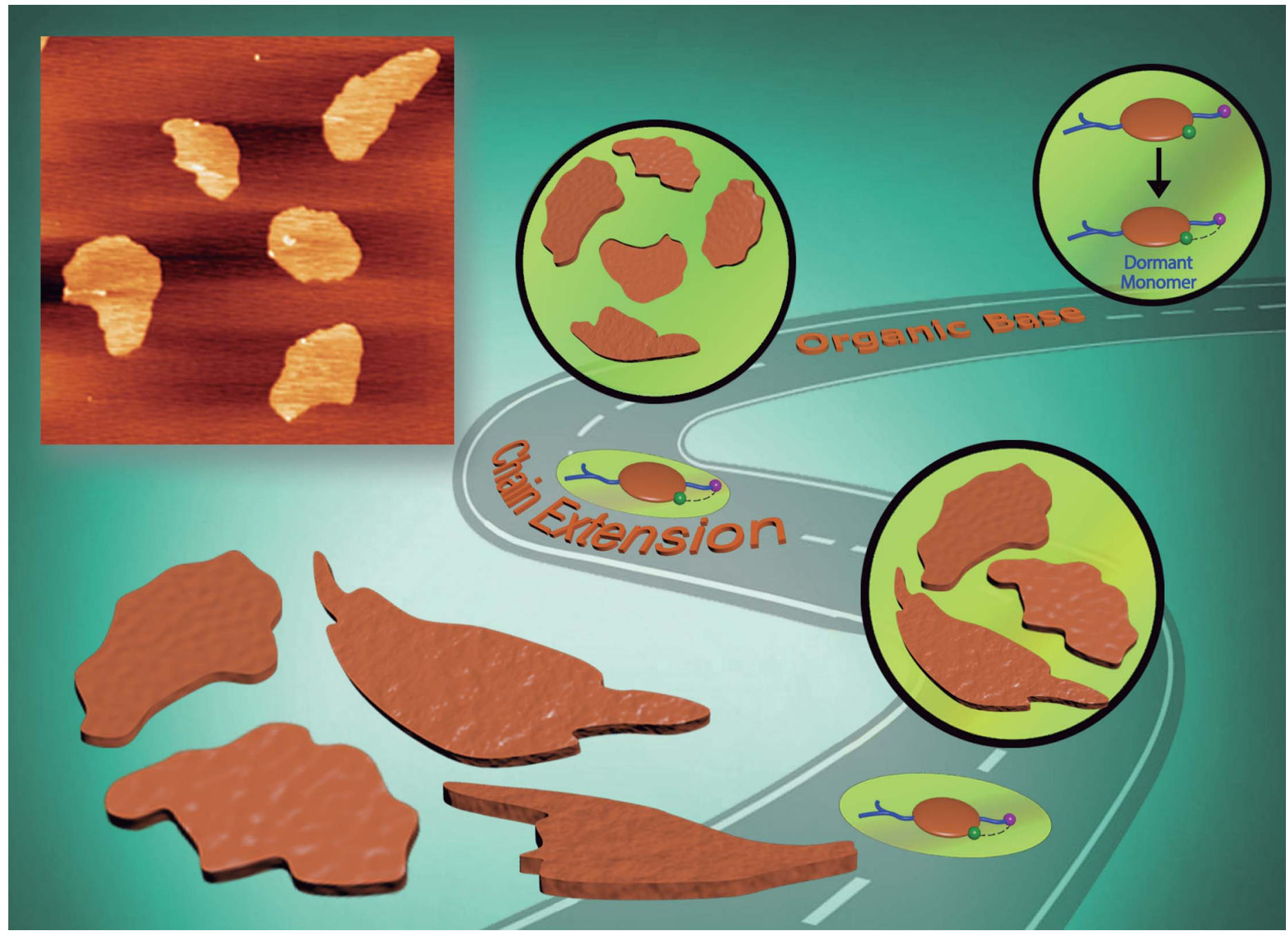

Showcasing research from Professor Suhrit Ghosh's laboratory, School of Applied and Interdisciplinary Sciences, Indian Association for the Cultivation of Science, Kolkata, India.

Organobase triggered controlled supramolecular ring opening polymerization and 2D assembly

This work elucidates organobase triggered living supramolecular polymerization of a naphthalene-diimide derivative containing a carboxylic acid. Under particular solution preparation conditions, supramolecular assembly by extended $\mathrm{H}$-bonding can be inhibited by intra-molecular $\mathrm{H}$-bonding between the carboxylic acid group and the adjacent carbonyl oxygen of the imide group. An organic base such as DMAP or DBU triggers controlled supramolecular polymerization of this dormant state leading to the formation of a well-defined 2D-assembly which can further act as a macro-initiator for chain extension and production of larger sheet.

\section{As featured in:}

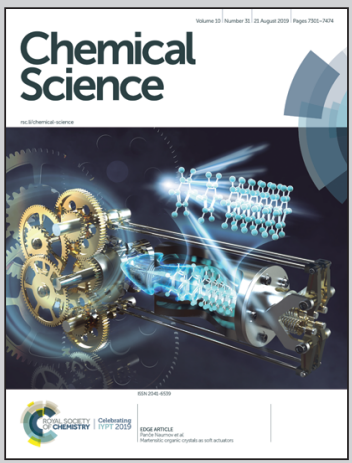

See Suhrit Ghosh et al.,

Chem. Sci., 2019, 10, 7345. 
Check for updates

Cite this: Chem. Sci., 2019, 10, 7345

( All publication charges for this article have been paid for by the Royal Society of Chemistry

\title{
Organobase triggered controlled supramolecular ring opening polymerization and 2D assembly $\dagger$
}

\author{
Anwesha Chakraborty, $\ddagger^{a}$ Goutam Ghosh, $t^{a}$ Deep Sankar Pal, ${ }^{a}$ Shinto Varghese ${ }^{b}$ \\ and Suhrit Ghosh (DD*a
}

A carboxylic acid appended naphthalene-diimide (NDI) derivative spontaneously aggregates in decane to generate a kinetically controlled product with irregular fibrillar morphology. By fine-tuning the sample preparation conditions, the carboxylic acid group can be trapped by intra-molecular $\mathrm{H}$-bonds with the adjacent imide carbonyl, which retards the spontaneous aggregation. In the presence of a catalytic amount of a non-nucleophilic organic base (DBU or DMAP), the meta-stable monomer exhibits supramolecular polymerization through a thermodynamically controlled pathway involving simultaneous $\mathrm{H}$-bonding and $\pi$-stacking and generates ultra-thin 2D nano-sheets. DMAP/DBU helps in ring-opening of the intra-molecularly $\mathrm{H}$-bonded monomer and in situ breeds the free acid, which, beyond a critical concentration, initiates controlled supramolecular ring opening polymerization (SROP) via the chaingrowth mechanism. The 2D polymer acts as a macro-initiator for subsequent two cycles of SROP and produces laterally extended ultra-thin nano-sheets.

Received 20th April 2019

Accepted 26th June 2019

DOI: $10.1039 / \mathrm{c} 9 \mathrm{sc} 01972 \mathrm{c}$

rsc.li/chemical-science

more recently with $\pi$-conjugated small molecules. ${ }^{6}$ However, the seed, which essentially plays a similar role to that of an

\section{Introduction}

Controlled supramolecular polymerization (CSP) ${ }^{1}$ has emerged as an active research area in the recent past. CSP emulates controlled/living covalent chain polymerization and endows control over the degree of polymerization, dispersity and successful synthesis of block copolymers. Rather counterintuitively, supramolecular polymers, ${ }^{2}$ albeit having an internal order, ${ }^{3}$ lack the structural precision in the mesoscopic scale. As exemplified in the recent literature, pathway complexity in supramolecular polymerization ${ }^{4}$ produces distinctly different products from the same building block depending on the conditions. This provides a clue for designing strategies for supramolecular polymerization by the chain growth mechanism. For example, a monomer, under kinetic control, generates polymer P1 while under thermodynamic control produces P2. P2 can be fragmented to form a seed, which guides the pool of monomers, trapped in the P1 state, to polymerize in a chain growth fashion with features similar to controlled/living covalent polymerization. Such possibilities have been successfully demonstrated in crystallization driven living supramolecular polymerization of macromolecules ${ }^{5}$ and

${ }^{a}$ School of Applied and Interdisciplinary Science, $2 A$ and $2 B$ Raja S. C. Mullick Road, Kolkata, India-700032. E-mail: psusg2@iacs.res.in

${ }^{b}$ Technical Research Center, Indian Association for the Cultivation of Science, $2 A$ and $2 B$ Raja S. C. Mullick Road, Kolkata, India-700032

$\dagger$ Electronic supplementary information (ESI) available: Synthesis of NDI derivatives, dormant monomer preparation, AFM and some additional experimental observations. See DOI: 10.1039/c9sc01972c

\$ These authors contributed equally. initiator in covalent chain growth polymerization, is not a discrete molecular entity, rather it is an ill-defined fragment of a pre-formed polymer. To best of our knowledge till date there is only one report ${ }^{6 a}$ demonstrating the use of a discrete molecular entity to trigger CSP. We have recently reported CSP of NDI-4 (Fig. 1a) by a seeding approach. ${ }^{7}$ In methyl-cyclohexane, it showed spontaneous J-aggregation by inter-molecular $\mathrm{H}$ bonding among the carboxylic acid groups. However, by

a)

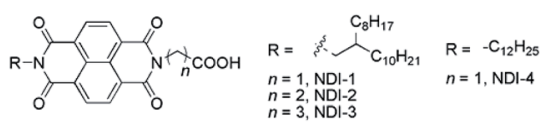

b)
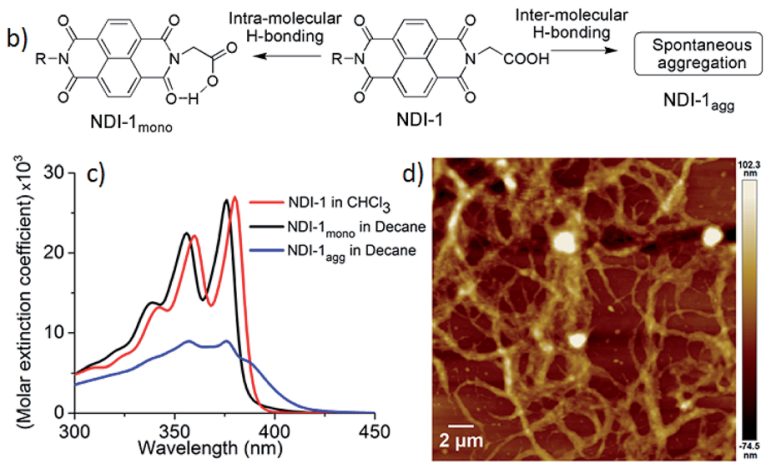

Fig. 1 (a) Structure of various NDI-derivatives; (b) Schematic showing pathway diversity in assembly of NDI-1; (c) Solvent dependent UV/Vis spectra of NDI-1 in monomeric and aggregated states; (d) AFM image of NDI- $1_{\text {agg }}$ in decane $\left[C=1 \times 10^{-4} \mathrm{M}\right]$. 
adopting a different solution preparation method, it was possible to isolate a meta-stable dormant state wherein the carboxylic acid groups were engaged in intra-molecular $\mathrm{H}$ bonding with the adjacent imide carbonyl group (Fig. 1b). The seed prepared from the J-aggregate was shown to be effective in initiating CSP of this dormant monomer producing a 1D polymer with tunable length. We envisaged that this particular system might offer an opportunity to explore CSP in the presence of an organobase (similar to organo-catalytic ring opening polymerization of a cyclic lactide or carbonate monomers) instead of a seed, if the organobase could open up the intramolecularly $\mathrm{H}$-bonded cyclic monomer by being a stronger $\mathrm{H}$ bond acceptor than the imide carbonyl. To test such possibilities, we have synthesized NDI-1 (Fig. 1a) which is structurally similar to previously reported NDI-4 (Fig. 1a), except for the peripheral alkyl chains. As control molecules, NDI-2 and NDI-3 (Fig. 1a) were also studied in which the intra-molecularly $\mathrm{H}$ bonded dormant state is anticipated to be relatively less stable due to larger ring size. In this article we reveal supramolecular ring opening polymerization (SROP) of NDI-1 in the presence of a catalytic amount of 1,8-diazabicyclo[5.4.0] undec7-ene (DBU) or 4-dimethylaminopyridine (DMAP) producing well defined 2D sheets with controllable dimensions in contrast to the uncontrolled fibrillar network structure produced by the spontaneous aggregation.

\section{Results and discussion}

The UV/Vis spectrum of NDI-1 in $\mathrm{CHCl}_{3}$ (Fig. 1c) exhibits sharp absorption bands with a vibronic fine structure which is typical of monomeric dyes. ${ }^{8}$ However, in a hydrocarbon solvent decane, which is conducive for $\mathrm{H}$-bonding, a broad structure-less absorption band with reduced intensity indicates ill-defined aggregates (NDI- $1_{\text {agg }}$ ) with a critical aggregation concentration (CAC) of $3 \times 10^{-5} \mathrm{M}$ (Fig. S1 $\dagger$ ). The AFM image (Fig. 1d) in decane reveals the formation of an irregular fibrillar network for NDI$1_{\text {agg }}$. Our previously reported $\operatorname{method}^{7}$ allowed isolating the monomeric state of NDI-4 only at a very dilute concentration $(1 \times$ $10^{-5} \mathrm{M}$ ). To improve on that we have now introduced a branched alkyl chain into NDI-1 to reduce its propensity for spontaneous aggregation. Indeed, NDI- $1_{\text {mono }}$ could be isolated at ten times higher concentration $\left(1 \times 10^{-4} \mathrm{M}\right)$ than NDI- $4_{\text {mono }}$ in decane by slightly modified sample preparation conditions. Instead of directly dissolving NDI-1 in decane, it was dissolved in $\mathrm{CHCl}_{3}$ / $\mathrm{MeOH}$ and dried to form a thin film. It was then dissolved in $\mathrm{CHCl}_{3}$ and an aliquot was mixed with decane and then the good solvent was selectively removed by evaporation. The UV/Vis spectrum of the so-prepared sample in decane was found to be almost identical to that in $\mathrm{CHCl}_{3}$ (Fig. 1c) except for a blue shift due to the solvatochromic effect which confirmed formation of the monomeric state (NDI- $1_{\text {mono }}$ ) in decane. The fluorescence spectrum of NDI- $1_{\text {mono }}$ in decane appeared similar (Fig. S2 $\dagger$ ) to that in $\mathrm{CHCl}_{3}$ reconfirming lack of aggregation. In contrast the fluorescence spectrum of NDI- $1_{\text {agg }}$ clearly showed a broad excimer peak (Fig. S2 $\dagger$ ). FT-IR spectra (Fig. S3†) of NDI-1 in $\mathrm{CHCl}_{3}$ showed the $\mathrm{O}-\mathrm{H}$ stretching band at $3087 \mathrm{~cm}^{-1}$ indicating non- $\mathrm{H}-$ bonded $\mathrm{COOH}$. For NDI- $1_{\text {agg }}$, the peak appeared at $2995 \mathrm{~cm}^{-1}$ suggesting intermolecular $\mathrm{H}$-bonding among the $\mathrm{COOH}$ groups in the aggregated state. ${ }^{6 \boldsymbol{b}}$ Interestingly for $\mathrm{NDI}-\mathbf{1}_{\text {mono, }}$, the $\mathrm{OH}$ stretching peak appeared at a different position $\left(3053 \mathrm{~cm}^{-1}\right)$ which is assigned to the intra-molecular H-bonding with the imide carbonyl (Fig. 1b). The $\mathrm{C}=\mathrm{O}$ stretching band of the carboxylic acid group for NDI-1 $1_{\text {agg }}$ in decane appeared (Fig. S3b $\dagger$ ) at $1732 \mathrm{~cm}^{-1}$, matching with that for NDI-1 in solid state indicating inter-molecular $\mathrm{H}$-bonding. However for $\mathrm{NDI}-\mathbf{1}_{\text {mono }}$ in decane, the band appeared at a higher wavenumber $\left(1761 \mathrm{~cm}^{-1}\right)$, indicating the $\mathrm{C}=\mathrm{O}$ of the $\mathrm{COOH}$ group is not involved in $\mathrm{H}$ bonding as should be the case for the proposed cyclic structure (Fig. 1b). To further support the formation of an intra-molecular $\mathrm{H}$-bonded state in NDI- $\mathbf{1}_{\text {mono }}$, control molecules NDI-2 and NDI-3 (Fig. 1a) were studied. In these cases also, the monomeric state could be trapped in decane following a similar sample preparation procedure. But the UV/Vis spectra of NDI- $2_{\text {mono }}$ and NDI-

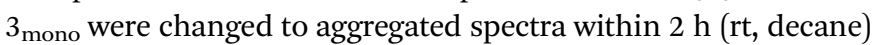
while the monomeric spectrum of NDI- $1_{\text {mono }}$ remained stable at least for $24 \mathrm{~h}$ at $\mathrm{rt}$ (Fig. $\mathrm{S} 4 \dagger$ ). ${ }^{9}$ This may be attributed to the increase in ring-size and further supports the hypothesis on the intra-molecularly $\mathrm{H}$-bonded structure of the monomeric state. To gain more insight, dispersion-corrected density functional theory (DFT-D3BJ) at the B3LYP/6-311G (d, p) level was employed for the optimization of the intra-molecular $\mathrm{H}$-bonded structures using Gaussian 16 program. ${ }^{10 a}$ Non-Covalent Interaction (NCI) analyses were used to get an estimate of the attractive and repulsive interactions. ${ }^{10 b}$ The reduced gradient density (RDG) isosurface gives a direct visualization of the real space interactions and the scattering plot of RDG against the sign of the second Hessian eigenvalue times the electron density $\left(\operatorname{sign}\left(\lambda_{2}\right) \rho\right)$ discriminates the attractive and the repulsive interactions as implemented in Multiwfn 3.6 software. ${ }^{10 c}$ The scattering plot (Fig. S5†) showed intra-molecular H-bond strength to be highest in NDI-1 while slightly lower in NDI-2 and considerably low in NDI-3 which corroborate with the experimental results.

Supramolecular ring opening polymerization (SROP) of NDI-

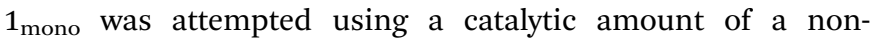
nucleophilic base DBU which has been extensively used for ring opening polymerization of cyclic lactides. ${ }^{\mathbf{1 1}}$ A solution of DBU (10 mol\%) in decane was added to a freshly prepared solution of NDI- $1_{\text {mono }}\left(1 \times 10^{-4} \mathrm{M}\right)$ in decane and supramolecular polymerization was monitored by UV/Vis spectroscopy (Fig. 2a) at $25{ }^{\circ} \mathrm{C}$. For the initial few minutes, no significant change was noticed, but after about $35 \mathrm{~min}$, the band intensity started decreasing sharply followed by saturation in about $75 \mathrm{~min}$. The mole fraction of the aggregate $\left(\alpha_{\mathrm{agg}}\right)$ (for calculations see ESI $\dagger$ ) $v s$. time plot (inset-Fig. 2a) revealed an initial lag phase prior to the onset of the supramolecular polymerization. Intriguingly, the UV-Vis spectra of the DBU triggered supramolecular polymer $\left(\mathrm{NDI}-1_{\mathrm{SP}}\right)$ and $\mathrm{NDI}-1_{\mathrm{agg}}$ were distinctly different. It appears that for NDI- $1_{\mathrm{SP}}$ there was a face to face $\pi$ stacking with rotational displacement along the long axis ${ }^{8}$ resulting in the appearance of a low-energy shoulder band unlike the broad structure-less spectrum for NDI- $1_{\text {agg }}$ (Fig. 1b).

The AFM image of NDI- $1_{\text {SP }}$ showed (Fig. $2 b$ ) a well defined 2D uniform nanosheet ${ }^{12}$ morphology with height and diameter in the range of $2.0 \mathrm{~nm}$ and $400 \mathrm{~nm}$, respectively, in sharp contrast to 
a) $\stackrel{2}{x}$

b)
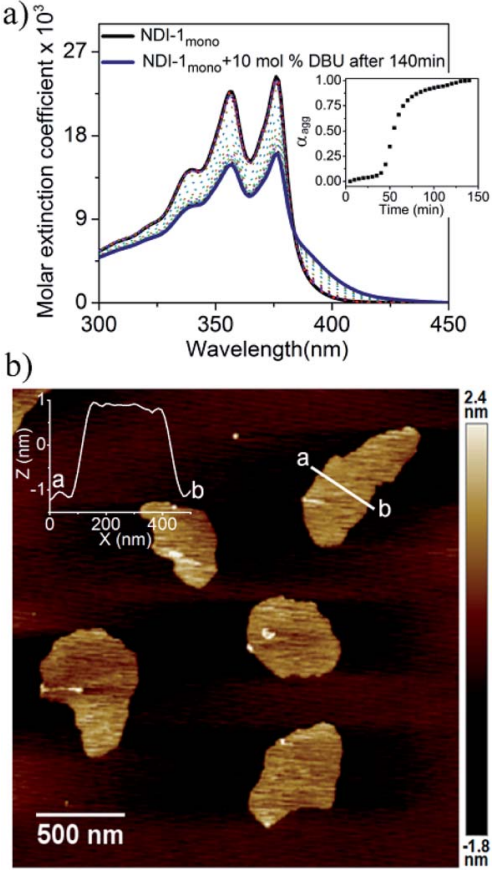

Fig. 2 (a) Time dependent change in the UV/Vis spectra of NDI- $1_{\text {mono }}$ in the presence of $10 \mathrm{~mol} \% \mathrm{DBU}$ in decane. Inset - variation of $\alpha_{\text {agg }}$ (estimated from the change in $A_{376} \mathrm{~nm}$ ) as a function of time; (b) AFM

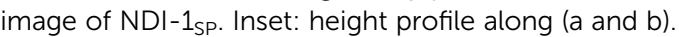

the irregular fibrillar network of NDI- $1_{\text {agg }}$ (Fig. 1c). Concentration dependent UV/Vis experiments in decane (Fig. 3a) revealed a significant difference in the CAC of NDI- $1_{\text {agg }}$ and NDI- $1_{\text {SP. }}$.

The ratio of absorption intensities at 375 and $356 \mathrm{~nm}$ has been used in the literature to probe $\pi$-stacking among the NDI chromophores. ${ }^{8}$ For NDI- $1_{\text {agg }}$, the ratio sharply increases below a critical concentration $\left(3 \times 10^{-5} \mathrm{M}\right)$ indicating disassembly while for NDI- $1_{\mathrm{SP}}$, no sign of disassembly was noticed even at $0.002 \mathrm{mM}$ indicating its much lower CAC. Furthermore, variable temperature UV/Vis studies (Fig. 3b, S6 $\dagger$ ) showed the onset of disassembly for NDI- $\mathbf{1}_{\text {agg }}$ just above $\mathrm{rt}$ and complete melting at $\sim 60{ }^{\circ} \mathrm{C}$, while for NDI- $1_{\mathrm{SP}}$, no disassembly was noticed up to $95{ }^{\circ} \mathrm{C}$. Therefore it is evident that DBU triggered CSP of NDI$1_{\text {mono guided the formation of a distinctly different product }}$
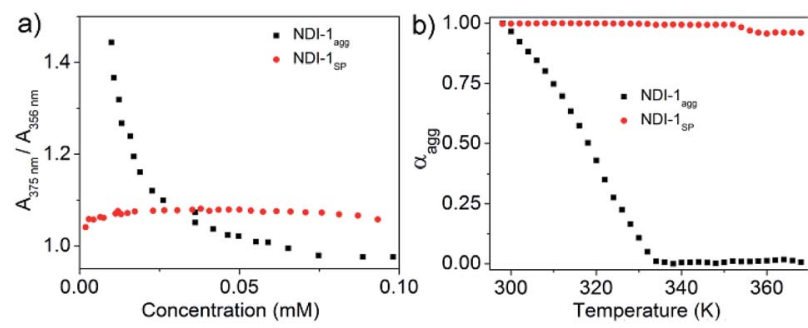

Fig. 3 Comparison of the (a) CAC and (b) thermal stability of NDI- $1_{\text {agg }}$ (aggregated spontaneously) and NDI-1 sP (prepared by CSP of NDI$1_{\text {mono }}$ using $10 \%$ DBU). $A_{375}$ and $A_{356}$ indicate absorption at 375 and $356 \mathrm{~nm}$, respectively, in the UV/Vis spectra in decane $[l=1 \mathrm{~cm} ; C=1 \times$ $10^{-4} \mathrm{M}$; $\alpha_{\text {agg }}$ at each temperature was calculated from the variable temperature UV/Vis spectra (Fig. S5 $†$ ).
$\left(\mathrm{NDI}-1_{\mathrm{SP}}\right)$ that not only differed in morphology but also exhibited a much higher stability and lower CAC than NDI- $\mathbf{1}_{\text {agg. To }}$ examine the generalized applicability of the method, SROP was also attempted using another commonly used organobase DMAP under identical conditions.

In this case, similar changes were noticed in the UV/Vis spectra (Fig. S7†) except that the lag phase was significantly reduced (Table 1). As the supramolecular polymerization was relatively faster in this case, DMAP was used as the base for all subsequent mechanistic investigations. SP was performed with varying amounts of DMAP and it was noticed that with increasing the concentration of the base (2.5 to $10 \mathrm{~mol} \%$ ), the initial lag phase shortened from $70 \mathrm{~min}$ to $20 \mathrm{~min}$ (Table 1 , Fig. S8 $\dagger$ ). AFM images showed formation of $2 \mathrm{D}$ nanosheets in all cases (Fig. 4b, S9†) with consistent height ( $\sim 2 \mathrm{~nm})$, similar to that observed for DBU triggered SROP. The size and aspect ratio of the sheets increased gradually with increasing amounts of DMAP (Table 1, Fig. S9†).

In a control experiment, a mixture of NDI-1 (not NDI- $1_{\text {mono }}$ ) and DMAP (10 mol\%) was dissolved in decane under hot conditions and the solution was allowed to equilibrate at rt for a couple of hours. This particular sample when examined under AFM, revealed a micrometer long entangled fibrillar network (Fig. 4a), in sharp contrast to the distinct 2D sheets obtained by SROP of NDI- $1_{\text {mono }}$ with the same amount of base (Fig. $4 \mathrm{~b}$ ). The height of the fibers was found to be $>20 \mathrm{~nm}$ which is much larger than $2 \mathrm{~nm}$ thick nanosheets. Therefore it is confirmed that the mere presence of DMAP was not responsible for the formation of $2 \mathrm{D}$ sheets, but trapping the active monomer in the dormant state was essential to realize the base triggered SROP. Not to mention that NDI- $1_{\text {mono }}$ alone did not produce $2 \mathrm{D}$ sheets either (Fig. S4†) after seven days confirming the essential role played by the organobase in the SROP. It is also noteworthy that NDI-2 or NDI-3, in which case isolation of a stable dormant monomeric state was not possible, also did not produce $2 \mathrm{D}$ sheets in the presence of DMAP, rather produced ill-defined aggregates or fibrillar morphology (Fig. S10†). To test whether the methodology is applicable to another monomer, DMAP triggered SROP was tested with NDI-4 which differs from NDI-1 in the structure of the peripheral alkyl chains. By the modified sample preparation method, NDI-4 also was successfully trapped in the monomeric state in decane at $C=10^{-4} \mathrm{M}$ (Fig. S11a $\dagger$ ). In the presence of 10\% DMAP, a lag phase followed by supramolecular polymerization was noticed (Fig. S11b $\dagger$ ) similar to that observed for NDI- $1_{\text {mono. The AFM image }}$ (Fig. S11c $\dagger$ ) confirmed formation of 2D sheets identical to that produced by NDI- $1_{\text {mono }}$. It is noteworthy that NDI-4 mono when polymerized using the seed (prepared from its spontaneously formed J-aggregate) produced J-aggregates and 1D fiber as evident from the UV/Vis spectrum and AFM image (Fig. S12 $\dagger$ ). ${ }^{7}$

The same monomer now when polymerized using DMAP revealed different modes of $\pi$-stacking as evident from the absence of any J-band and 2D nanostructure (Fig. S11a-c $\dagger$ ), indicating that the DMAP triggered SROP indeed followed a different pathway than seeded supramolecular polymerization. 
Table 1 Various parameters related to SROP of NDI-1 mono $_{\text {and }}$ resulting 2D nano-sheets

\begin{tabular}{lcccc}
\hline Base & mol\% & Lag time $^{a}(\mathrm{~min})$ & Height $^{b}(\mathrm{~nm})$ & Area $^{b}\left(\mu \mathrm{m}^{2}\right)$ \\
\hline DBU & 10 & 35 & 2.0 & 0.19 \\
DMAP & 10 & 20 & 2.0 & 0.12 \\
DMAP & 5.0 & 40 & 2.0 & 0.08 \\
DMAP & 2.5 & 2.0 & 0.03 & 1.6 \\
${ }^{a}$ Calculated from UV/Vis. ${ }^{b}$ Calculated from AFM images using ImageJ software (values reported are average of 10 sheets).
\end{tabular}

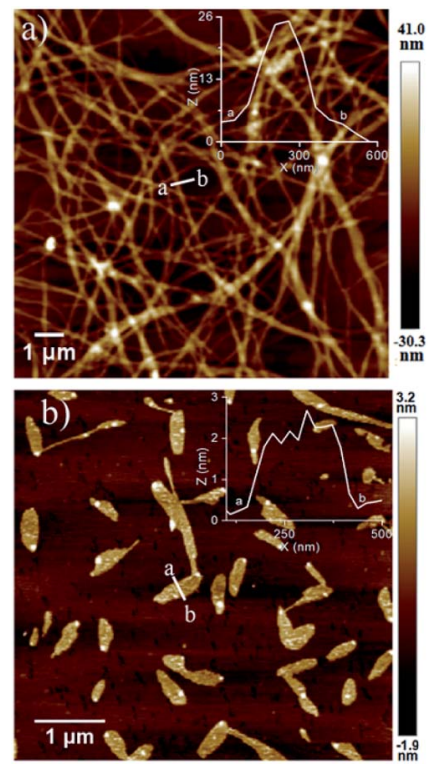

Fig. 4 AFM images of (a) a spontaneous supramolecular polymer produced by NDI-1 in the presence of 10 mol\% DMAP and (b) NDI-1 produced by SROP of NDI- $1_{\text {mono }}$ in the presence of 10 mol\% DMAP. In both cases, samples were drop cast on the mica surface at the end of polymerization $\left[\mathrm{C}=1 \times 10^{-4} \mathrm{M}\right]$.

Having established the distinct supramolecular polymerization pathway for $\mathrm{NDI}-1_{\text {mono }} / \mathrm{NDI}-4_{\text {mono }}$ in the presence of an organobase, we sought to examine the mechanistic details of the SROP. What role does DBU/DMAP play in the SROP? Is it an initiator or a catalyst? In chain growth polymerization, increasing the initiator/monomer ratio should decrease the degree of polymerization. Therefore the reverse trend (Table 1, Fig. S8 $\dagger$ ) observed for DMAP triggered SROP of NDI- $1_{\text {mono }}$ eliminates the role of DMAP as an initiator. It is proposed (Scheme 1) that DBU/DMAP, being a stronger H-bond acceptor than carbonyl oxygen, opens the intra-molecularly $\mathrm{H}$-bonded ring to produce the intermediate 1 which remains in equilibrium with the free acid. This is evident from the FT-IR spectra (Fig. S13†) which show a characteristic shift in the $\mathrm{C}=\mathrm{N}$ stretching frequency of DMAP indicating its involvement in $\mathrm{H}$ bonding. The lag phase observed in all cases possibly corresponds to the time required to establish the equilibrium and reaching a threshold concentration of the free acid beyond which it is able to form a nucleus (either by associating with itself or with the monomer) that subsequently initiates SROP of NDI- $1_{\text {mono }}$ via the chain growth mechanism. The lag phase was

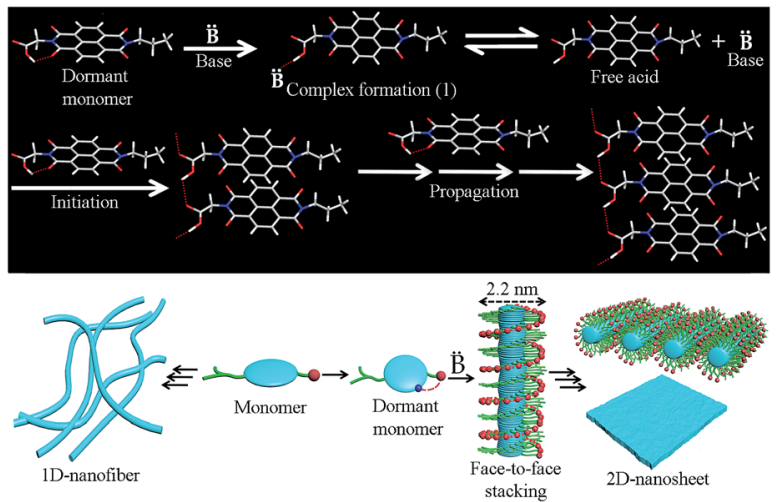

Scheme 1 Top - probable mechanistic pathway for organobase triggered SROP of NDI-1mono; bottom - pathway diversity and 2Dassembly of the product produced by SROP.

found to increase with increasing temperature and at $35^{\circ} \mathrm{C}$, the supramolecular polymerization did not begin even after $6 \mathrm{~h}$ (Fig. S14†). This is conceivable because two of the three steps before propagation include non-covalent association which in organic solvent is likely to be driven by enthalpy and thereby should be less pronounced at elevated temperature. DBU being a stronger base than DMAP, the equilibrium remains more shifted towards the intermediate 1 (Scheme 1) and therefore it requires longer time to attain the critical free acid concentration for nucleation and thus the lag phase is longer. ${ }^{13}$

On the other hand, with increasing concentration of DMAP, it takes less time to generate the required amount of free acid for initiation of SROP and therefore the lag phase shortens. Increase in the size of the sheets with increasing DMAP concentration may be correlated with increase in monomer conversion, which in fact was evident by comparing the UV/Vis spectra of the end products (Fig. S15 $\dagger$ ) in three different DMAPtriggered SROP. If indeed this mechanism is operating, then the free acid itself should be able to initiate the polymerization. To test this possibility, a solution of NDI-1 $\left(C=1 \times 10^{-5} \mathrm{M}<\mathrm{CAC}\right.$ of NDI- $1_{\text {agg }}$ ) in decane was added to the NDI- $1_{\text {mono }}$ solution and indeed it could initiate the SROP leading to the formation of similar 2D nanosheets (Fig. S16 $\dagger$ ). It is noteworthy that the CAC of NDI-1 $1_{\mathrm{SP}} \ll$ NDI- $1_{\text {agg }}$ (Fig. 3 ) and thus nucleation for NDI-1 $1_{\mathrm{SP}}$ at $0.01 \mathrm{mM}\left(<\mathrm{CAC}\right.$ of NDI- $\left.1_{\text {agg }}\right)$ is conceivable.

Interestingly in this case, no lag phase was noticed (Fig. S16 $\dagger$ ) which further supports the hypothesis that the time taken to reach a critical concentration of the free acid for nucleation is probably responsible for the observed lag phase in DMAP/DBU triggered supramolecular polymerization. From the 
sharp difference in the UV/Vis spectra and AFM images of NDI$1_{\text {agg }}$ and $\mathrm{NDI}-1_{\mathrm{SP}}$, it is clear that they represent distinctly different structures. Interestingly, when the NDI- $1_{\text {agg }}$ solution was heated up to $90{ }^{\circ} \mathrm{C}$ and cooled slowly $\left(1 \mathrm{~K} \mathrm{~min}^{-1}\right)$, the UV/ Vis spectrum (Fig. S17†) did not look similar to the initial spectrum, rather it exhibited similar features to those observed for the SROP suggesting that NDI- $1_{\text {agg }}$ is a kinetically controlled product whereas the thermodynamically controlled product was formed during SROP (Scheme 2).

This is further evident by the contrasting melting curves of NDI- $1_{\text {agg }}$ and NDI- $1_{\text {SP }}$ (Fig. 3). It is believed that such pathway diversity originates from different possible $\mathrm{H}$-bonding motifs of the $\mathrm{COOH}$ group $^{\mathbf{1 4}}$ in NDI-1 which includes (i) syn-syn catemer type extended chain or (ii) dimerization followed by $\pi$-stacking in the orthogonal direction or (iii) simultaneous $\pi$-stacking and $\mathrm{H}$ bonding by stacking of the monomer units on top of each. Previous reports from our group and others ${ }^{15}$ showed prominent J-aggregation in the case of syn-syn catemer type H-bonding driven assembly of structurally similar building blocks.

Therefore, the lack of such a prominent J-band in the UV/Vis spectra of either NDI- $1_{\text {agg }}$ or $\mathrm{NDI}-1_{\mathrm{SP}}$ eliminates the possibility of packing in mode (i). Important to note that the height of the nano-sheets is slightly higher than the length of NDI-1 but lower than its dimer length which supports the packing in mode (iii) for SROP. It is believed that DAMP triggered SROP leads to the formation of a $1 \mathrm{D}$ structure by simultaneous $\pi$-stacking and $\mathrm{H}$ bonding, which by lateral clustering produces $2 \mathrm{D}$ sheets. In fact, during the AFM imaging of a sample before completion of the polymerization, co-existence of such $1 \mathrm{D}$ rod-like structures and incompletely grown 2D sheets could be seen (Fig. 5). Interestingly, the heights of the individual rods and the $2 \mathrm{D}$ sheets were found to be exactly identical which supports the fact that the sheets are indeed built by the assembly of these rods. On the other hand, for NDI-1 agg, mode (ii) appears to be mainly operating in which case less stability is conceivable as in this mode $\mathrm{H}$-bonding and $\tau$-stacking do not enjoy a synergistic effect. The difference in the mode of $\mathrm{H}$-bonding between NDI- $\mathbf{1}_{\text {agg }}$ and $\mathrm{NDI}-\mathbf{1}_{\text {sp }}$ was evident from the FT-IR spectra (Fig. S18 $\dagger$ ) which showed a clear difference in the position of the peak corresponding to the $\mathrm{O}-\mathrm{H}$ stretching of the carboxylic acid group.

Finally the living character of the 2D supramolecular polymer generated by SROP of NDI- $1_{\text {mono }}$ was investigated by UV/Vis

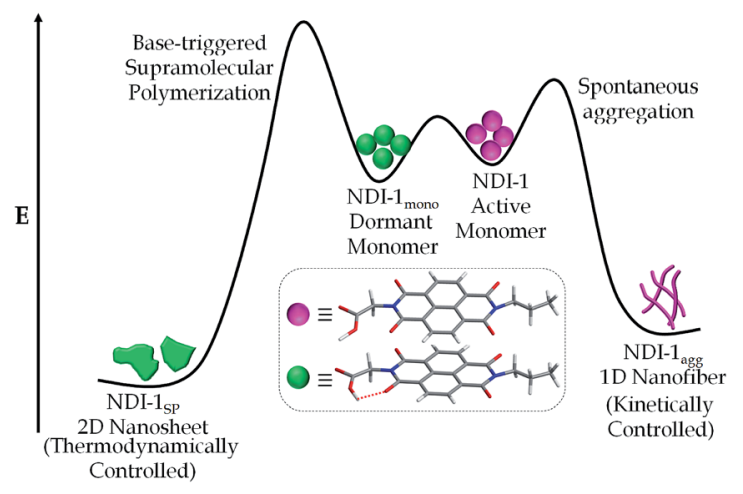

Scheme 2 Pathway complexity in supramolecular assembly of NDI-1.
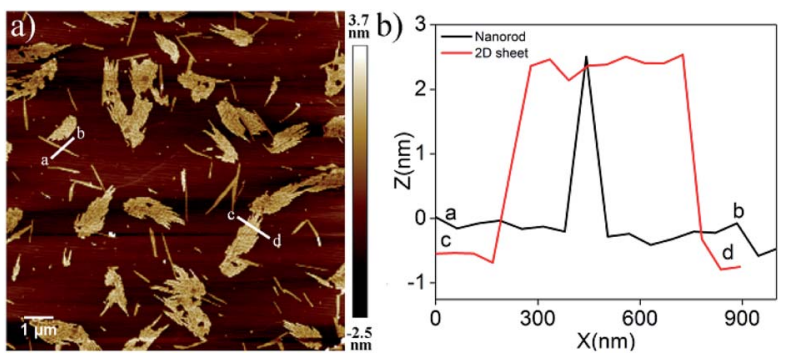

Fig. 5 (a) AFM height image of a sample prepared from the solution taken out from the 10\% DMAP triggered supramolecular polymerization vessel (before completion of the polymerization) showing coexistence of rods and sheets; (b) height profile (across $a-b$ and $c-d$ in the image) of nano-rods and $2 \mathrm{D}$ sheets $\left[C=1 \times 10^{-4} \mathrm{M}\right]$.

spectroscopy and AFM. ${ }^{\boldsymbol{b}, \boldsymbol{d}, \boldsymbol{g}}$ Initially a fixed quantity of DMAP $(2.5 \mathrm{~mol} \%)$ was added to NDI- $1_{\text {mono }}$ solution $(1.5 \mathrm{ml}, C=1 \times$ $10^{-4} \mathrm{M}$ ) which triggered SROP after a lag phase (Fig. 6, S19 $\dagger$ ) and was completed in $\sim 3 \mathrm{~h}$. AFM images (Fig. 7a) showed formation of uniform nanosheets with a height and width of approximately $2 \mathrm{~nm}$ and $150 \mathrm{~nm}$, respectively.

An aliquot of the so-prepared $\mathrm{NDI}-1_{\mathrm{sp}}$ solution was transferred to a cuvette containing another batch of freshly prepared NDI- $1_{\text {mono }}$ solution $\left(\mathrm{NDI}-1_{\text {mono }} / \mathrm{NDI}-1_{\text {sp }}=34\right.$ ) while polymerization started immediately without any lag phase and the reaction was over within $2 \mathrm{~h}$ (Fig. 6). The AFM image showed (Fig. 7b) significant growth of the nano-sheets (height $\sim 2 \mathrm{~nm}$, width/area $\sim 305 \mathrm{~nm} / 0.18{\mu \mathrm{m}^{2}}^{2}$ ) indicating that the initially formed sheets indeed served the role of a macro-initiator for subsequent SROP and chain extension.

It is important to note that the prominent lag phase that is observed in the first cycle is absent in the second cycle indicating that unlike DMAP (during first cycle), initially formed nano-sheets acted as an initiator. In fact the nano-sheet produced after $2^{\text {nd }}$ cycle was also found to be active for initiating the SROP in the $3^{\text {rd }}$ cycle (again without any lag phase) resulting in further chain extension. The AFM image showed (Fig. 7c) formation of much larger sheets (height $\sim 2 \mathrm{~nm}$, width/ area $\sim 540 \mathrm{~nm} / 0.74 \mu^{2}$ ) without compromising with the uniformity of the structure.

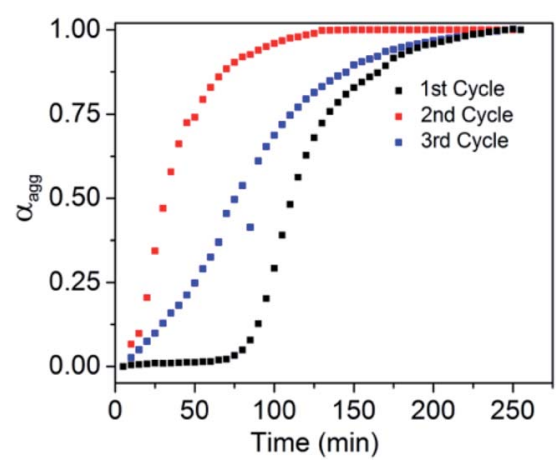

Fig. 6 Variation of $\alpha_{\text {agg }}$ Vs. time for living SROP; 2.5\% DMAP was used in the first cycle while NDI-1 $1_{\mathrm{SP}}\left(\mathrm{NDI}-1_{\text {mono }} / \mathrm{NDI}-1_{\mathrm{SP}}=34\right)$ was used in the $2^{\text {nd }}$ and $3^{\text {rd }}$ cycles. 

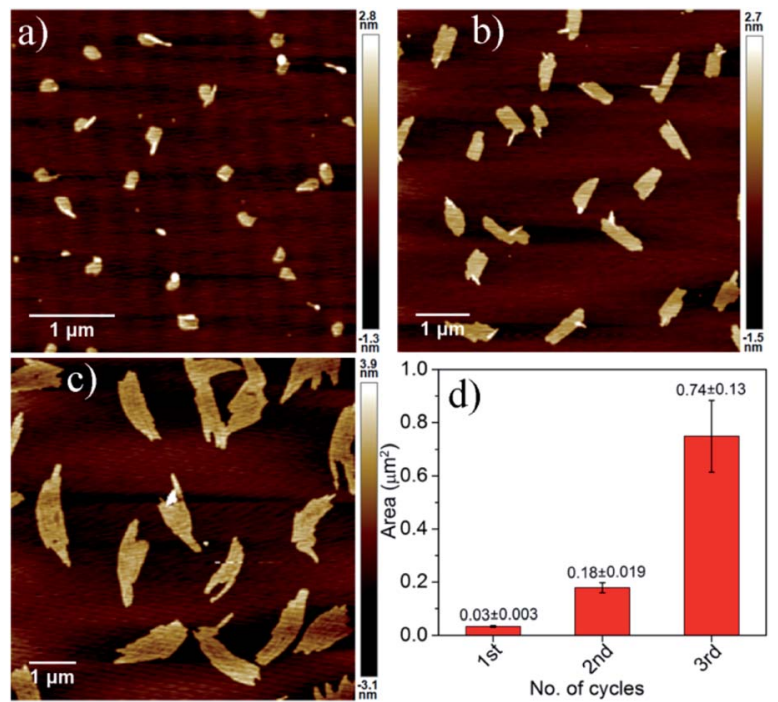

Fig. 7 AFM images of NDI-1 $1_{\text {SP }}$ after (a) first, (b) second and (c) third cycles of living SROP and (d) their average area.

\section{Conclusions}

Overall we have demonstrated an unprecedented example of controlled supramolecular ring opening polymerization, triggered by a well-known organic base. Although pathway complexity and seeded supramolecular polymerization have been fairly well known now, in most examples neither the initiator (seed) nor the starting material (kinetically controlled aggregate) is a molecularly defined entity. In a significant step forward, both are discrete molecules in the present design. That opens up future opportunities for extending the design to wide ranging $\mathrm{p}$ - and n-type semiconductors of different electronic properties and also synthesis of supramolecular copolymers with segregated blocks of two different semiconductors. ${ }^{16}$ Such structures should be of significant importance, particularly considering the rarely reported (in the context of supramolecular assembly of $\pi$-systems) ultra-thin $2 \mathrm{D}$ morphology ${ }^{12}$ which is in the limelight because of the unusual physical properties resulting from the quantum size effect of the ultra-thin structure. ${ }^{17}$ Further investigation on the mechanistic details, possibility of employing other $\mathrm{H}$-bond donor/acceptor molecules for SROP,$^{18}$ general applicability of the methodology for other $\pi$ systems and understanding the impact of 2D-nanostructure on photo-physical properties ${ }^{19}$ are underway in our laboratory.

\section{Conflicts of interest}

There are no conflicts to declare.

\section{Acknowledgements}

AC thanks the DST-Inspire Program for a research fellowship. GG thanks IACS for a research fellowship. SV thanks the TRC,
IACS for funding. SG thanks DST, India, for funding (SwarnaJayanti Fellowship : DST/SJF/CSA-01/2-14-15).

\section{Notes and references}

1 (a) F. Würthner, Nat. Chem., 2014, 6, 171; (b) R. D. Mukhopadhyay and A. Ajayaghosh, Science, 2015, 349, 241; (c) D. van der Zwaag, T. F. A de Greef and E. W. Meijer, Angew. Chem., Int. Ed., 2015, 54, 8334; (d) B. Adelizzi, N. J. V. Zee, L. N. J. de Windt, A. R. A. Palmans and E. W. Meijer, J. Am. Chem. Soc., 2019, 141, 6110.

2 (a) L. Brunsveld, B. J. B. Folmer, E. W. Meijer and R. P. Sijbesma, Chem. Rev., 2001, 12, 4071; (b) Z. Chen, A. Lohr, C. R. Saha-Möller and F. Würthner, Chem. Soc. Rev., 2009, 38, 564; (c) S. S. Babu, V. K. Praveen and A. Ajayaghosh, Chem. Rev., 2014, 114, 1973; (d) E. Krieg, M. M. C. Bastings, P. Besenius and B. Rybtchinski, Chem. Rev., 2016, 4, 2414; (e) L. Yang, X. Tan, Z. Wang and X. Zhang, Chem. Rev., 2015, 115, 7196; (f) C. Rest, R. Kandanellia and G. Fernández, Chem. Soc. Rev., 2015, 44, 2543.

3 T. Aida, E. W. Meijer and S. Stupp, Science, 2012, 335, 813. 4 P. A. Korevaar, T. F. A. de Greef and E. W. Meijer, Chem. Mater., 2014, 26, 576.

5 (a) C. E. Boott, J. Gwyther, R. L. Harniman, D. W. Hayward and I. Manners, Nat. Chem., 2017, 9, 785; (b) N. Petzetakis, A. P Dove and R. K. O'Reilly, Chem. Sci., 2011, 2, 955.

6 (a) J. Kang, D. Miyajima, T. Mori, Y. Inoue, Y. Itoh and T. Aida, Science, 2015, 347, 646; (b) S. Ogi, K. Sugiyasu, S. Manna, S. Samitsu and M. Takeuchi, Nat. Chem., 2014, 6, 188; (c) S. Ogi, V. Stepanenko, K. Sugiyasu, M. Takeuchi and F. Würthner, J. Am. Chem. Soc., 2015, 137, 3300; (d) A. Pal, M. Malakoutikhah, G. Leonetti, M. Tezcan, M. Colomb-Delsuc, V. D. Nguyen, J. van der Gucht and S. Otto, Angew. Chem., Int. Ed., 2015, 54, 7852; (e) S. Ogi, T. Fukui, M. L. Jue, M. Takeuchi and K. Sugiyasu, Angew. Chem., Int. Ed., 2014, 53, 14363; (f) W. Wagner, M. Wehner, V. Stepanenko, S. Ogi and F. Würthner, Angew. Chem., Int. Ed., 2017, 56, 16008; (g) S. Ogi, K. Matsumoto and S. Yamaguchi, Angew. Chem., Int. Ed., 2018, 57, 2339; (h) E. E. Greciano, B. Matarranz and L. Sánchez, Angew. Chem., Int. Ed., 2018, 57, 4697; (i) J. S. Valera, R. Gómez and L. Sánchez, Angew. Chem., Int. Ed., 2019, 58, 510; (j) E. E. Greciano and L. Sánchez, Chem.-Eur. J., 2016, 22, 13724; (k) J. S. Valera, R. Gómez and L. Sánchez, Small, 2018, 14, 1702437.

7 D. S. Pal, H. Kar and S. Ghosh, Chem. Commun., 2018, 54, 928.

8 (a) M. A. Kobaisi, S. V. Bhosale, K. Latham, A. M. Raynor and S. V. Bhosale, Chem. Rev., 2016, 116, 11685; (b) A. Das and S. Ghosh, Chem. Commun., 2016, 52, 6860.

9 The fate of NDI- $1_{\text {mono }}$ in decane was monitored for seven days by UV/Vis spectroscopy and AFM (Fig. S4†) in the absence of any added organobase. The monomeric state was stable for $24 \mathrm{~h}$, but subsequently some precipitation was noticed which was also evident by the increase in the baseline intensity of the UV/Vis spectra due to scattering. 
The AFM image of the sample prepared after seven days showed the presence of heterogeneous spherical particles.

10 (a) M. J. Frisch, et. al., Gaussian 16, Revision B.01, Gaussian, Inc., Wallingford CT, 2016; (b) E. R. Johnson, S. Keinan, P. Mori-Sánchez, J. Contreras-García, A. J. Cohen and W. Yang, J. Am. Chem. Soc., 2010, 132, 6498; (c) T. Lu and F. Chen, J. Comput. Chem., 2012, 33, 580.

11 N. E. Kamber, W. Jeong and R. M. Waymouth, Chem. Rev., 2007, 107, 5813.

12 (a) T. Fukui, S. Kawai, S. Fujinuma, Y. Matsushita, T. Yasuda, T. Sakurai, S. Seki, M. Takeuchi and K. Sugiyasu, Nat. Chem., 2017, 9, 493; (b) C. E. Boott, A. Nazemi and I. Manners, Angew. Chem., Int. Ed., 2015, 54, 13876; (c) M. Vybornyi, A. Rudnev and R. Häner, Chem. Mater., 2015, 27, 1426; (d) M. Pfeffermann, R. Dong, R. Graf, W. Zajaczkowski, T. Gorelik, W. Pisula, A. Narita, K. Müllen and X. Feng, J. Am. Chem. Soc., 2015, 137, 14525; (e) S. Ghosh, D. S. Philips, S. Saeki and A. Ajayaghosh, Adv. Mater., 2017, 29, 1605408.
13 D. Vacognea Charlotte and H. Schlaad, Chem. Commun., 2015, 51, 15645.

14 J. N Moorthy, J. Indian Inst. Sci., 2008, 88, 131.

15 (a) M. R. Molla, D. Gehrig, L. Roy, V. Kamm, A. Paul, F. Laquai and S. Ghosh, Chem.-Eur. J., 2014, 20, 760; (b) F. Salerno, J. A. Berrocal, A. T. Haedler, F. Zinna, E. W. Meijer and L. D. Bari, J. Mater. Chem. C, 2017, 5, 3609.

16 B. Adelizzi, A. Aloi, A. J. Markvoort, H. M. M. T. Eikelder, I. K. Voets, A. R. A. Palmans and E. W. Meijer, J. Am. Chem. Soc., 2018, 140, 7168.

17 A. K. Geim and K. S. Novoslev, Nat. Mater., 2006, 6, 183.

18 Preliminary investigations were carried out to examine the possible role of $\mathrm{H}$-bonding donors such as TFA or pyrrole in initiating SROP. In the presence of TFA, no sign of supramolecular polymerization was noticed (Fig. S20 †) after $6 \mathrm{~h}$. But pyrrole could initiate supramolecular polymerization with relatively shorter lag phase producing 2D sheets (Fig. S21†).

19 S. K. Park, J. H. Kim and S. Y. Park, Adv. Mater., 2018, 30, 1704759. 